\title{
Re-Challenge with Clozapine after Neuroleptic Malignant Syndrome and Seizure in a Patient with Di-George Syndrome: Case Report and Review of Literature
}

\author{
Geetha Chandrashekar ${ }^{1 *}$, Ganesh Gopalakrishna ${ }^{2}$, Austin Campbell ${ }^{3}$, Katherine Edwards ${ }^{1}$, \\ Muaid Ithman' ${ }^{1}$
}

${ }^{1}$ Department of Psychiatry, University of Missouri, Columbia, USA

${ }^{2}$ Department Psychiatry, University of Arizona, Tucson, USA

${ }^{3}$ Department of Pharmacy, University of Missouri Health Care, Columbia, USA

Email: *chandrashekarg@health.missouri.edu

How to cite this paper: Chandrashekar, G., Gopalakrishna, G., Campbell, A., Edwards, K. and Ithman, M. (2020) Re-Challenge with Clozapine after Neuroleptic Malignant Syndrome and Seizure in a Patient with Di-George Syndrome: Case Report and Review of Literature. Open Journal of Psychiatry, 10, 9-14.

https://doi.org/10.4236/ojpsych.2020.101002

Received: November 22, 2019

Accepted: December 20, 2019

Published: December 23, 2019

Copyright $\odot 2020$ by author(s) and Scientific Research Publishing Inc. This work is licensed under the Creative Commons Attribution International License (CC BY 4.0).

http://creativecommons.org/licenses/by/4.0/

\begin{abstract}
Background: Individuals with 22q11.2DS, a genetic subtype of Schizophrenia, respond as well to clozapine as those with other forms of Schizophrenia. It has been reported that serious and rare adverse events like seizures, and myocarditis have been associated with clozapine treatment in this population. To the best of our knowledge, the incidence of neuroleptic malignant syndrome (NMS) as an adverse effect of antipsychotic use in patients with this disorder has not yet been reported. Aim: In this article, we discuss a case of clozapine-induced NMS and subsequent re-challenge in a patient with 22q11.2DSassociated schizophrenia. The aim of this study is to accumulate scientific data about rare presentations, and serve as a major educational tool, and highlight the unique challenges faced when using clozapine in a patient with $\mathrm{Di}$ George Syndrome. Methods: This is a descriptive case report of a patient encountered in the inpatient unit which includes retrospective review of the patient's electronic medical record and a literature review of antipsychotic medications-induced NMS. Conclusion: This study demonstrates a successful re-challenge with clozapine after the patient developed NMS and seizures during the initial treatment and also highlights how, in addition to drug level monitoring, considering pharmacogenetic testing early in treatment might help minimize adverse drug reactions in individuals with known genetic disorders such as $22 \mathrm{q} 11.2 \mathrm{DS}$.
\end{abstract}

\section{Keywords}

Clozapine, DiGeorge Syndrome, 22q11.2 Deletion Syndrome, Neuroleptic 
Malignant Syndrome (NMS), Seizure, Re-Challenge

\section{Introduction}

Chromosome 22q11.2 deletion syndrome (22q11.2DS), also known as DiGeorge syndrome, is a rare genetic disorder estimated to occur in roughly 1 in 4000 live births [1]. Presentations of the disorder vary greatly, but often include clinical features such as cardiac abnormalities, immunodeficiency, cleft palate, and hypocalcemia. In addition to the many medical complications, nearly one third of individuals with 22q11.2DS will develop schizophrenia or schizoaffective disorder [2]. To date there are no formal guidelines outlining treatment of 22q11.2DS related schizophrenia, resulting in symptomatic treatment with standard treatment protocols. Limited data would suggests response rates are similar to control groups, however, patients with 22q11.2DS related schizophrenia appear to have greater likelihood of experiencing serious adverse effects, such as seizures or neutropenia [2] [3].

Neuroleptic malignant syndrome (NMS) is an uncommon but life-threatening complication of exposure to antipsychotics. It is estimated that $0.02 \%-0.03 \%$ of patients exposed to antipsychotics developed NMS with a mortality of 5.6\% [4]. Individuals characteristically present with symptoms of hyperthermia, muscle rigidity, mental status changes, and autonomic instability. While a small number of risk factors do exist, the occurrence of NMS is still considered to be idiosyncratic and unpredictable [5]. To date, there have been no reports of NMS experienced by patients with 22q11.2DS related schizophrenia. This article talks about a case of clozapine-induced NMS and subsequent re-challenge in a patient with 22q11.2DS-associated schizophrenia.

\section{Case Presentation}

A 25-year-old Caucasian male with moderate intellectual disability, and DiGeorge syndrome was admitted to an acute psychiatric hospital for worsening aggression, auditory and visual hallucinations, and suicidal and homicidal ideations. History revealed that he was first diagnosed with schizophrenia at age 15, and had been previously admitted multiple times for psychosis and aggressive behaviors in different child and adult psychiatric facilities. He was diagnosed with DiGeorge syndrome at age 16 after a genetic testing that revealed 22q11.2 deletion.

At the time of admission, his medication regimen consisted of three different antipsychotics (fluphenazine $5 \mathrm{mg}$ four times daily, olanzapine $25 \mathrm{mg}$ at bedtime, and quetiapine $500 \mathrm{mg}$ at bedtime), two benzodiazepines (clonazepam 1 mg three times daily and temazepam $30 \mathrm{mg}$ at bedtime), one antidepressant (citalopram $10 \mathrm{mg}$ daily), and one mood stabilizer (divalproex ER $500 \mathrm{mg}$ every morning and $1000 \mathrm{mg}$ every evening). History revealed that he had previously 
tried risperidone, aripiprazole, carbamazepine, fluvoxamine, and venlafaxine. Despite these multiple medications, the patient continued to have refractory psychotic symptoms and worsening aggression and had become an imminent harm to himself and others. Given that he had tried and failed multiple antipsychotic medications in the past, our plan was to minimize polypharmacy and transition him to clozapine during this hospitalization.

In the first week of admission, quetiapine and fluphenazine tapers were initiated and olanzapine was rapidly tapered off and discontinued. Upon olanzapine discontinuation, clozapine was commenced and gradually titrated up by 25 $\mathrm{mg} /$ day. The citalopram and both benzodiazepines were more recent additions prior to the patient's admission, however the rational for their use was unclear. Therefore citalopram and temazepam were discontinued, and a clonazepam taper initiated. Depakote was continued to help with mood stability, and for seizure prophylaxis given the increased propensity of seizures in this population and that clozapine could further lower seizure threshold. Benztropine was continued for extrapyramidal symptoms with a plan to taper off at outpatient.

In the following week, quetiapine and clonazepam were successfully tapered off and discontinued. Clozapine was progressively increased to a dose of $100 \mathrm{mg}$ every morning and $200 \mathrm{mg}$ at bedtime. Fluphenazine was continued at a lower dose with plan to taper it slowly as an outpatient, given that the patient endorsed some auditory hallucinations following rapid dose reductions. The patient continued to have auditory and visual hallucinations but had no significant physical aggression. The patient was planned to be discharged back to his ISL after 16 days of treatment with further dose adjustments planned as an outpatient.

However, the night prior to this planned discharge, the patient developed a fever (up to 40.7 degrees Celsius) and hypertension (145/82 initially). Creatinine kinase $(\mathrm{CK})$ was found to be high $(788 \rightarrow 1152$ units/L). Acute mental status changes and motor abnormalities were difficult to be appreciated given patient's baseline tremor and intellectual disability. Neuroleptic malignant syndrome was suspected and patient was transferred to medical services where he remained for one week. All psychotropic medications were held. A thorough workup for hyperthermia including urinalysis, chest X-ray, blood cultures, and a lower extremity ultrasound, were negative. A lumbar puncture did not demonstrate any abnormalities.

After he returned to the psychiatric unit, the plan was to re-challenge with clozapine after a 14 day washout period. However within that first week, he demonstrated poor oral intake, significant apathy and subsequent vomiting which resulted in a transfer back to the medical unit. During his second stay on the medical unit, he experienced a tonic-clonic seizure. Divalproex ER was restarted at this time. After being stabilized, the patient was readmitted to the psychiatry unit. The treatment team had an extended discussion with his parents about the risk of recurrent NMS, recurrent seizures, other adverse effects, prognosis, and options available for treatment. They were also counseled about the 
risks and benefits of re-challenge with clozapine after the NMS and seizures.

After careful consideration of the various options for treating his psychosis, the patient was restarted on clozapine. The slow titration with a $12.5 \mathrm{mg}$ increase every other day was planned and carried out during the next four weeks of his stay in the psychiatric hospital. At the time of discharge, the patient was on a total dose of $137.5 \mathrm{mg}$ daily. Prior to discharge, the patient appeared brighter, and was better able to participate in the interview. Clozapine levels were drawn at steady state and indicated the patient was a slow metabolizer of the medication (total clozapine $=408$, clozapine $=319$, and norclozapine $=89$ ). We also obtained a baseline $\mathrm{T}$-cell count for monitoring in the future.

After discharge the patient was scheduled for followed up in our outpatient clinic. He continues to do well on clozapine and has not had any recurrence of NMS or seizure-like episodes. The caregivers continue to report marked improvement in his mood and his social interaction with others. He has not demonstrated any physical aggression, and has not required any further hospitalizations.

\section{Discussion}

Approximately $1 \%$ of patients with schizophrenia also have 22q11.2DS. As compared to schizophrenia in the general population, these patients have similar prognosis, clinical features, and neurocognitive profiles [2]. There is limited evidence of using clozapine in this subtype of schizophrenia. Aksu et al. reported the successful use of clozapine in a 15-year-old girl with 22q11.2DS and improvement of Clinical Global Impressions-Severity (CGI-S) score [6]. A retrospective study to determine the long-term safety and efficacy of clozapine compared 20 patients with schizophrenia and 22q11.2DS to 20 patients with schizophrenia matched for age and clinical severity [3]. Despite sample size limitations of the study, it found both groups had similar clinical responses to clozapine and reduction in hospitalizations, however, patients with 22q11.2DS were more prone to serious adverse events, like seizures. Diagnosis of NMS in this subtype is often complicated by the clinical features of DiGeorge syndrome. Intellectual disability, common in DiGeorge syndrome [7], and motor abnormalities confounds the assessment for acute mental status changes and muscular rigidity. Also, hypocalcemia is a laboratory anomaly found in NMS and an inherent feature of DiGeorge syndrome due to hypoparathyroidism.

Limited evidence exists about re-challenging patients with clozapine after the development of NMS [8]. With the risk of recurrence of NMS being as high as $30 \%$, prescribers typically consider re-challenge with a different antipsychotic [4]. This may not be ideal in patients determined to have treatment-resistant schizophrenia, where clozapine is the only FDA approved treatment option.

This patient was particularly challenging for a multitude of reasons. The clinical course and prognosis was complicated with the development of NMS and a seizure episode. A definitive causal relationship between clozapine alone and NMS was unable to be established given the cornucopia of medications used in 
this case. However, using Naranjo Algorithm for Adverse Drug Reactions [9], it can be suggested that clozapine was a possible cause (score $=3$ ) given that NMS is a rare, but well recognized side effect of clozapine [10]; it followed a temporal sequence after clozapine was introduced, and that it resolved after withholding clozapine. Other possible causes include fluphenazine, seroquel, depakote [11] or a combination of all the medications. Furthermore, a pharmacogenetic testing was not done in this case prior to starting clozapine. He was later determined to be a slow metabolizer based on his clozapine and norclozapine levels. A prior pharmacogenetic testing would have informed dosing decisions and potentially prevented adverse drug reactions.

To our knowledge this is also the only report of NMS in patients with DiGeorge syndrome and a subsequent re-challenge with clozapine after NMS and seizures. DiGeorge syndrome provides unique challenges when treated with clozapine. Patients with DiGeorge syndrome have low lymphocyte counts due to thymic hypoplasia or aplasia. Despite neutropenia being a concerning adverse effect of clozapine, other hemopoietic lines can be at risk [12], placing these patients at particular risk for infections. Hence, in addition to regular laboratory monitoring, obtaining a baseline T-cell count for future monitoring may be of value in this population of patients.

\section{Conclusion}

Although 22q11.2DS is not a common co-morbid condition in schizophrenia, this sub-type deserves more attention as the extent of its genomic influence on the clinical portrait is significant but remains largely unclear. As such, there is limited evidence for using clozapine in patients with this particular subtype of schizophrenia. With this case report, we demonstrate the successful use of clozapine in such a patient through a re-challenge after the patient developed NMS and seizure during initial treatment. This case also illustrates how, in addition to drug level monitoring, considering pharmacogenetic testing early in treatment may be prudent to help minimize adverse drug reactions in individuals with known underlying genetic disorders such as 22q11.2DS.

\section{Conflicts of Interest}

The authors declare no conflicts of interest regarding the publication of this paper.

\section{References}

[1] Goodship, J., Cross, I., LiLing, J. and Wren, C. (1998) A Population Study of Chromosome 22q11 Deletions in Infancy. Archives of Disease in Childhood, 79, 348-351. https://doi.org/10.1136/adc.79.4.348

[2] Bassett, A.S. and Chow, E.W. (2008) Schizophrenia and 22q11.2 Deletion Syndrome. Current Psychiatry Reports, 10, 148-157. https://doi.org/10.1007/s11920-008-0026-1

[3] Butcher, N.J., Fung, W.L.A., Fitzpatrick, L., et al. (2015) Response to Clozapine in a Clinically Identifiable Subtype of Schizophrenia. British Journal of Psychiatry, 206, 
484-491. https://doi.org/10.1192/bjp.bp.114.151837

[4] Pileggi, D.J. and Cook, A.M. (2016) Neuroleptic Malignant Syndrome Review: Focus on Treatment and Rechallenge. Annals of Pharmacotherapy, 50, 973-981. https://doi.org/10.1177/1060028016657553

[5] Berman, B.D. (2011) Neuroleptic Malignant Syndrome, A Review for Neurohospitalists. The Neurohospitalist, 1, 41-47. https://doi.org/10.1177/1941875210386491

[6] Aksu, H. and Demirkaya, S.K. (2016) Treatment of Schizophrenia by Clozapine in an Adolescent Girl with DiGeorge Syndrome. Journal of Child and Adolescent Psychopharmacology, 26, 652. https://doi.org/10.1089/cap.2016.0044

[7] McDonald-McGinn, D.M., Emanuel, B.S. and Zackai, E.H. (1993) 22q11.2 Deletion Syndrome, in GeneReviews(R). In: Pagon, R.A., et al., Eds., Seattle.

[8] Anbalagan, E., Ithman, M. and Lauriello, J. (2014) Rechallenging Clozapine after Neuroleptic Malignant Syndrome. Psychiatric Quarterly, 85, 345-348. https://doi.org/10.1007/s11126-014-9291-9

[9] Naranjo, C.A., Busto, U., Sellers, E.M., Sandor, P., Ruiz, I., Roberts, E.A., et al. (1981) A Method for Estimating the Probability of Adverse Drug Reactions. Clinical Pharmacology \& Therapeutics, 30, 239-245. https://doi.org/10.1038/clpt.1981.154

[10] Karagianis, J.L., Phillips, L.C., Hogan, K.P. and LeDrew, K.K. (1999) ClozapineAssociated Neuroleptic Malignant Syndrome: Two New Cases and a Review of the Literature. Annals of Pharmacotherapy, 33, 623-630. https://doi.org/10.1345/aph.18286

[11] Yıldırım, V., Direk, M.Ç., Güneş, S., Okuyaz, Ç. and Toros, F. (2017) Neuroleptic Malignant Syndrome Associated with Valproate in an Adolescent. Clinical Psychopharmacology and Neuroscience, 15, 76-78. https://doi.org/10.9758/cpn.2017.15.1.76

[12] Assion, H.J., et al. (1996) Lymphocytopenia and Thrombocytopenia during Treatment with Risperidone or Clozapine. Pharmacopsychiatry, 29, 227-228.

https://doi.org/10.1055/s-2007-979577 